\title{
Study of FAE Explosive Image Analysis Based on the Fractional Dimension
}

\author{
Yucai Dong, Hongyan Li, Hongtao Shi \& lianghai Yi \\ Institute of Nonlinear Science \\ Academy of Armored Forces Engineering, Beijing 100072, China
}

\begin{abstract}
The method of box dimension is used in this paper for calculating the fractal dimension of the fuel air explosive(FAE) explosive images. The relationship between fractal dimension and the expansion law of fireball is discussed. The change of explosion image fractal dimension all the time is studied, and its change law is inferred, with the reason analyzed, which lays the foundation for the further study of explosive process.
\end{abstract}

Keywords: FAE, Fractal, Box Dimension, Cloud

\section{Introduction}

Fractal geometry is a non-linear science which studies irregular physique attribute by American nationality France mathematician B.B.Mandelbrot in 1973. As a result of its object of studying widespread, at present it goes thorough natural social sciences in each domain day by day, and develops unceasingly. Fractal has the following characteristics: Fractal geometry graph 's irregularity everywhere and different criterion graph's regularity, and the fractal dimension is bigger than the topology dimension.Fractal dimension plays important role in the fractal fundamental research, which is an important parameter in describing image fractal characteristic. Fractal dimension analysis of fractal image has the vital significance for the characteristic and rule of exploration fractal image change.

The detonation image can record the fuel physical disperser, characteristics of burning and detonation in detonation process, which can reflect each performance in the knocking fuel. At present the domestic and foreign research scholars have done massive work in method of researching the detonation image processing. Dong Yucai , in literature (Dong Yucai, 2007), introduces the fractal theory in the detonation image processing, calculates fractal dimension in the liquid fuel detonation process in various times using the box dimension method, summarizes the fractal dimension change rule in the liquid fuel detonation process, and analyzes the detonation spread change near field. Liu Gengran used the high speed colored camera in literature (Liu Gengran, 2007) to record process picture of $0.5 \mathrm{~kg}$ solid state FAE detonation diffusion. In experiment, camera's photography frequency is $2000 \mathrm{f} / \mathrm{s}$, each gap is $0.5 \mathrm{~ms}$. The picture size is $640 \times 480$, which is preserved by the JPG document format. This paper has calculated mean radius and the duration for the solid state air fuel (SFAE) detonation fireball, summarize dissemination rule for explosive shock wave. On the foundation of the above experimental result, using fractal theory and through processing experimental image of exploding the spread, this paper obtains the detonation image fractal curve, calculates dimension value of border curve in various times and the dimension change rule. Moreover, this paper has analyzed the reason for dimension change, which lays foundation for analyzing the denotation process.

\section{Computation for Dimension of Detonation Image Fractal}

\subsection{Extract detonation border curve}

This paper applies the MATLAB software to process detonation image, and obtains the detonation curve gradually. The detonation image extraction divides into six steps. 
First we will read the detonation image in procedure, then the image will be preserved by $480 \times 640 \times 3$ matrix.In order to be advantageous for processing, transforms the image to the gradation image preserved, by $480 \times 640$, where each element is a picture element spot and the different element value represents different brightness or the gradation level. The value 0 represents the black and the value 255 represents the white. That the value is bigger indicated the brightness of picture element in this position is higher. In order to eliminate the irrelevant picture element disturbance in the graph, we need remove the graph background, and carry on screening the graph matrix through the selection threshold value. The value in this paper is taken by 240 , the original graph transformation is two value charts, the graph matrix transformation is $480 \times 640$ binary matrices, where element only has 0 and 1 . The digital 0 represents the position picture whose element in original graph matrix is smaller than or equal to 240 , but digital 1 is bigger than 240 , which is the same to the gradation image. When demonstrating graph, the value 0 represents the black and the value 1 represents the white. While cutting the background image, this paper constitutes a matrix with the last image, and carries on each image to spot ride with this matrix, then the curve obtained is the boundary curve of detonation image.

The above is a detonation image in the $15 \mathrm{~ms}$ time. Figure (1) is the primitive image photographed, figure (2) is the gradation chart, figure (3) is two value charts, figure (4) is the initial boundary curves, figure (5) is the image removing the impurity background, figure (6) namely is the boundary diagram of curves finally obtained

\subsection{Calculate curve fractal dimension using box dimension}

Fractal dimension is the most core content in the fractal theory, which is discussed most much in the fractal theory. Also it is studied most widespread and can reflect the complex degree of fractal image. The computation methods of fractal dimension quite are many, where the box dimension is most commonly used.

Define box dimension: Supposes $A \subset R_{n}$ is a non-spatial set, under Euclid distance, $A$ is contained close with the small box for length of side $\delta$. Let $N(A)$ be the smallest box number which contains $A$. Then the fractal box dimension is:

$$
D=\lim _{\delta \rightarrow 0} \frac{\ln N(A)}{-\ln \delta}
$$

This paper uses MATLAB to carry on the programming. First read boundary curve in procedure, then the boundary curve will be preserved by a $480 \times 640$ binary matrix. Structure length of side with $r_{1}=1$ (picture element), cover the image with this check, judge whether the covered small square does contain the boundary curve, and record the check number $K_{1}$ containing the image. Then take the length of side $r_{2}=2$ and record the check number $K_{2}, \cdots \cdots$. Take the length of side $r_{n}=2 * r_{n-1}$ and record the check number $K_{n}$ containing the image. If $r_{n}>480$, withdraw the circulation, then we will obtain two groups of data $r_{1}, r_{2}, \cdots, r_{n}$ and $K_{1}, K_{2}, \cdots, K_{n}$, which are taken the logarithm and linearly fit. In the last the inverse of the fitted straight line slope is namely the fractal dimension of boundary curve.

\section{Fractal dimension change analysis}

Through contrasting the picture, flame brightness has changes from bright to dark, then to bright again in the detonation process. When exploding in $0.5 \mathrm{~ms}$, since the explosion products have extremely high pressure, the rate of the denotion fireball outward expansion is extremely quick, whose interior radius can achieve $4.9 \mathrm{~m}$ in the extremely short time. Along with further proliferation of the explosion product, the explosion center pressure drops rapidly, the fireball edge flame starts to extinguish and the fireball radius changes rapidly small. But in this time massive nondetonation product in explosion center starts to burn, the flame starts to change bright, and diffuses outward, fireball radius slow fill-out. When fireball burning finished, flame brightness starts to change dark until extinguishment.

Figure 5 is the change tendency of various frequently fractal dimension in the SFAE fuel detonation process.

The dimension value change may divide into two stages: One is rise stage for the dimension value, two is the dimension tends to the steady stage.

First stage is the rise stage for dimension. Under explosive shock wave function fuel is staved and diffuses outward. These staved explosion product starts burning while diffusing outward, and produces many small burning pellets in the detonation fireball edge; Meanwhile as a result of the explosion center pressure, the fireball inflates unceasingly distorts, thus making the fireball edge to be more and more complex and the corresponding curve dimension assumes escalation trend. 
Fractal dimension of the second stage tends to steady. After the fireball expands to the limit radius, the shockwave has already been separated from the fireball contact surface, alone disseminates outward. In this time no new detonation and burning in the fireball edge occur. The fireball maintains the original shape and the fractal dimension also tends to steady.

Overall, the fractal dimension may divide into the rise stage and the steady stage, but from partial condition, the detonation dimension continuously is in during, which is showed in the following chart:

The reason of this phenomenon is that in detonation process fuel proliferation explodes, which can produce some burning explosion in the detonation fireball edge. These products interior can produce pressure and inflate while detonating, which causes the detonation fireball edge to become complex and the fractal dimension to increase; Meanwhile as a result of the detonation fireball internal pressure, those diffused explosion product can be separated, until burning extinguishes, thus making the entire fractal dimension to have a drop value. In the entire detonation process, the detonation fireball edge can have many such products, therefore the fractal dimension can be in during the shake.

\section{Conclusion}

Through comparison and analysis, the tendency of fractal dimension change and the rule of boundary curve is similar, which can reflect the change tendency of the boundary curve. Researching the detonation image and analyzing fireball change rule of detonation process has laid certain foundation for further researching detonation image and analyzing fireball change rule of detonation process foundation.But when withdrawing the boundary curve, the method of processing the edge impurity need to further study, which can obtain the more accurate dimension value.

References Dong Yucai, (2007). Reseach about median surface stave shape based on liquid detonation disperser of the fractal dimension. Mathematics in practice and theory.

Liu Gengran, (2007). Experimental study on explosive shock wave dissemination rule of detonation solid state FAE . Technical consultation review.

Liu Gengran, (2006). Reseach on detonation characteristic and kill effect of a kind of high energy Yang Shushen, Shao Longyi. Image fractal dimension computation under MATLAB environment . Journal of CUMT Mining Science and Technology.

Xin Houwen. Fractal theory and application. Hefei: University of Science and Technology of China Presse

Zhang Jizhong, (1997). Fractal. Beijing: Tsinghua University press .

Zeng Wenqu \& Wang Xiangyang \& Liu Dan etc. (1993). Fractal theory and fractal computer simulation, Shenyang: Northeast University press

Table 1 . Number of check contained by boundary curves in time $15 \mathrm{~ms}$

\begin{tabular}{cccccccc}
\hline $\begin{array}{c}\text { Check } \\
\text { radius } r\end{array}$ & $\begin{array}{c}\text { check } \\
\text { quantity } K\end{array}$ & $\begin{array}{c}r \text { takes } \\
\text { logarithm }\end{array}$ & $\begin{array}{c}K \text { takes } \\
\text { logarithm }\end{array}$ & $\begin{array}{c}\text { Check } \\
\text { radius } r\end{array}$ & $\begin{array}{c}\text { check } \\
\text { quantity } K\end{array}$ & $\begin{array}{c}r \text { takes } \\
\text { logarithm }\end{array}$ & $\begin{array}{c}K \text { takes } \\
\text { logarithm }\end{array}$ \\
\hline 1 & 745 & 0 & 2.8722 & 32 & 25 & 1.5051 & 1.3979 \\
2 & 443 & 0.3010 & 2.6464 & 64 & 11 & 1.8062 & 1.0414 \\
4 & 242 & 0.6021 & 2.3838 & 128 & 5 & 2.1072 & 0.6990 \\
8 & 120 & 0.9031 & 2.0792 & 256 & 2 & 2.4082 & 0.3010 \\
16 & 55 & 1.2041 & 1.7404 & & & & \\
\hline
\end{tabular}


Table 2. Border curve dimension value from $150 \mathrm{~ms}$ to $163 \mathrm{~ms}$

\begin{tabular}{cccccccc}
\hline time & dimension & time & dimension & time & dimension & time & dimension \\
\hline 150 & 1.1341 & 153.5 & 1.1597 & 157 & 1.1684 & 160.5 & 1.1847 \\
150.5 & 1.1412 & 154 & 1.1625 & 157.5 & 1.1668 & 161 & 1.1874 \\
151 & 1.1445 & 154.5 & 1.1664 & 158 & 1.1716 & 161.5 & 1.1819 \\
151.5 & 1.1493 & 155 & 1.1649 & 158.5 & 1.1643 & 162 & 1.1888 \\
152 & 1.1516 & 155.5 & 1.1625 & 159 & 1.1739 & 162.5 & 1.1895 \\
152.5 & 1.1517 & 156 & 1.1678 & 159.5 & 1.1802 & 163 & 1.1922 \\
153 & 1.1594 & 156.5 & 1.1662 & 160 & 1.1876 & 163.5 & 1.1909 \\
\hline
\end{tabular}

Table 3. border curve dimension value from $250 \mathrm{~ms}$ to $263 \mathrm{~ms}$

\begin{tabular}{cccccccc}
\hline time & dimension & time & dimension & time & dimension & time & dimension \\
\hline 250 & 1.2856 & 253.5 & 1.2718 & 257 & 1.2748 & 260.5 & 1.2764 \\
250.5 & 1.2789 & 254 & 1.2748 & 257.5 & 1.2717 & 261 & 1.276 \\
251 & 1.2834 & 254.5 & 1.2772 & 258 & 1.2746 & 261.5 & 1.276 \\
251.5 & 1.2672 & 255 & 1.2763 & 258.5 & 1.2759 & 262 & 1.2761 \\
252 & 1.2727 & 255.5 & 1.2792 & 259 & 1.2723 & 262.5 & 1.2762 \\
252.5 & 1.2735 & 256 & 1.2757 & 259.5 & 1.2757 & 263 & 1.2778 \\
253 & 1.2769 & 256.5 & 1.2759 & 260 & 1.2786 & 263.5 & 1.2789 \\
\hline
\end{tabular}

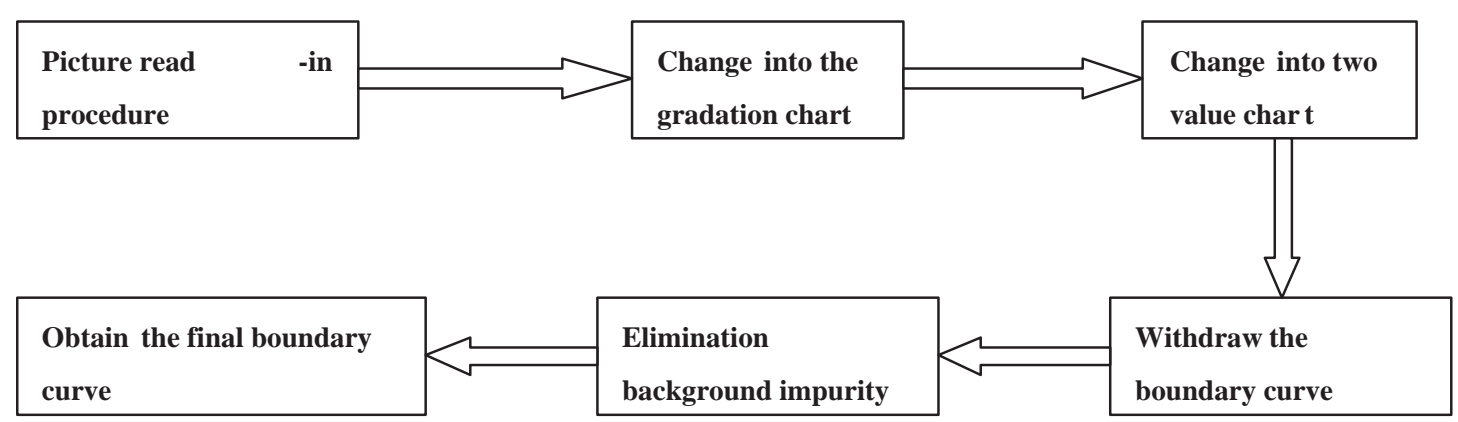

Figure 1. Flow chart for border curve extraction

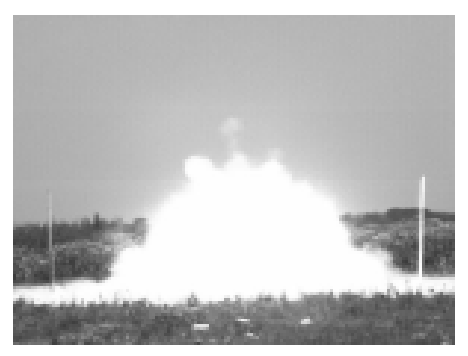

Figure (1)

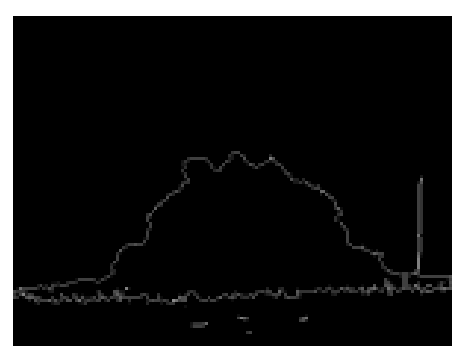

Figure (4)

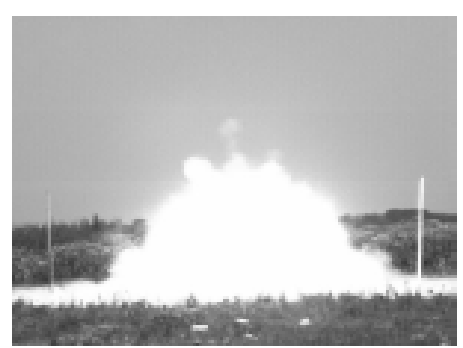

Figure (2)

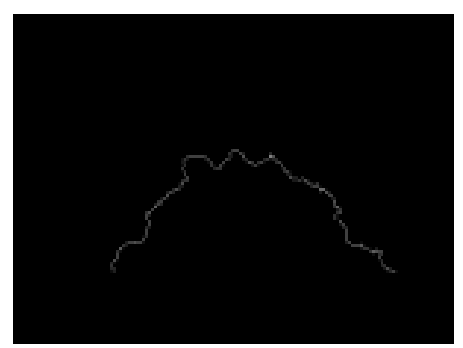

Figure (5)

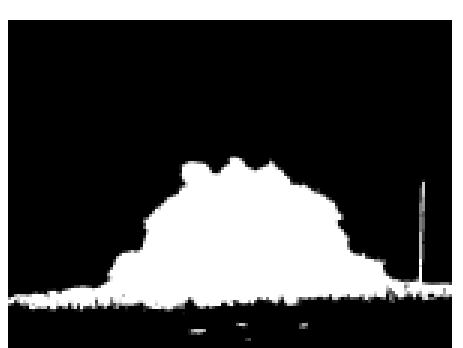

Figure (3)

Figure 2. Fitting chart for border curve fractal dimension in time $15 \mathrm{~ms}$ 


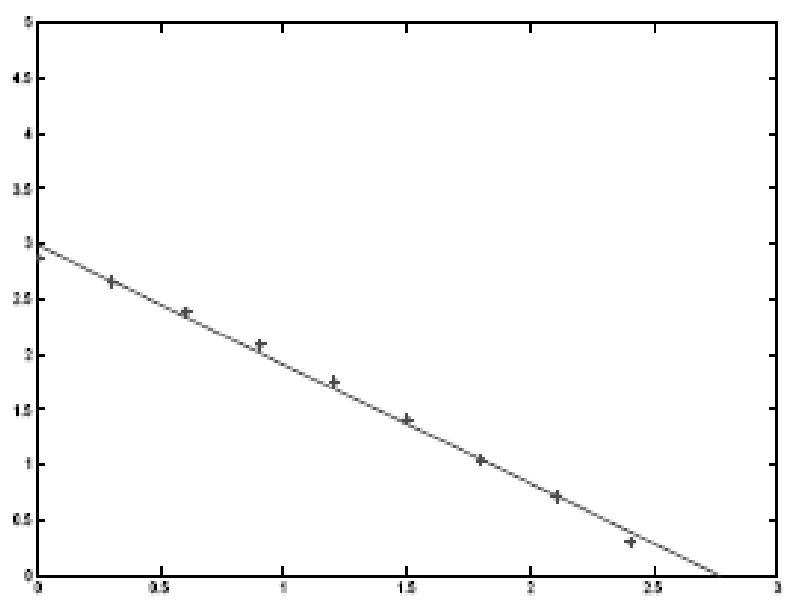

Figure 3. Fractal dimension fitting chart of the border curve in time $15 \mathrm{~ms}$

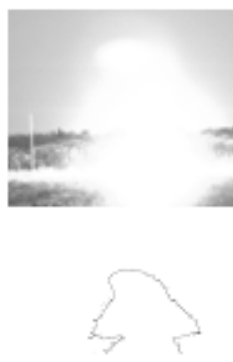

$0.5 \mathrm{~ms}$
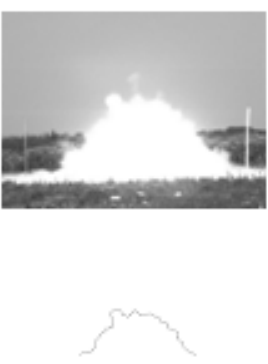

$10 \mathrm{~ms}$

Figure 4. Detonation chart and corresponding boundary of solid state FAE
$20 \mathrm{~ms}$

$30 \mathrm{~ms}$
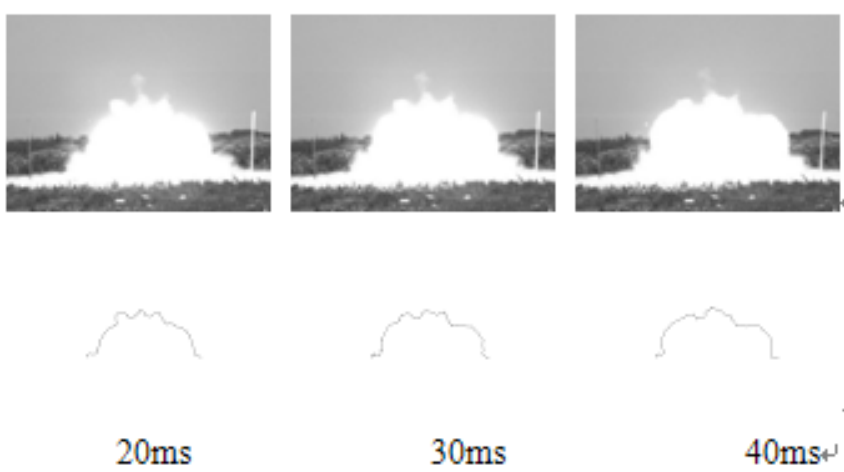

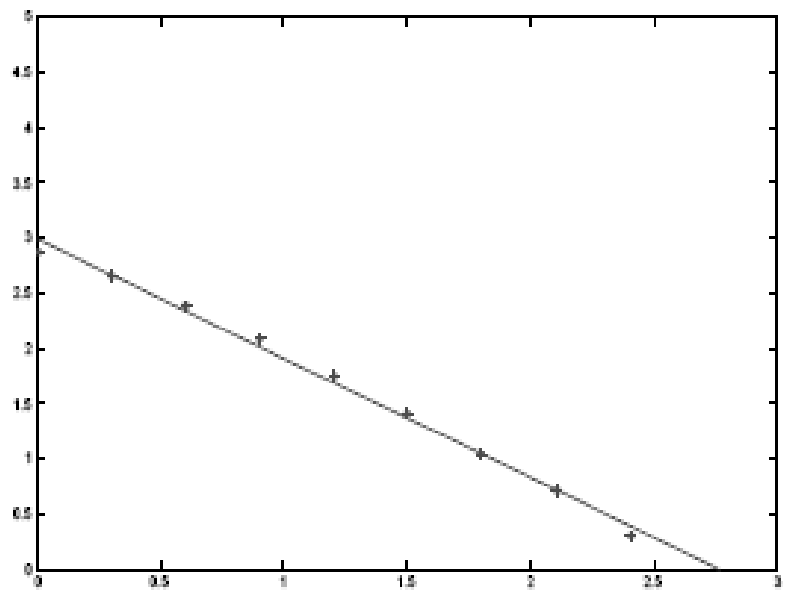

Figure 5. Fractal dimension change chart 


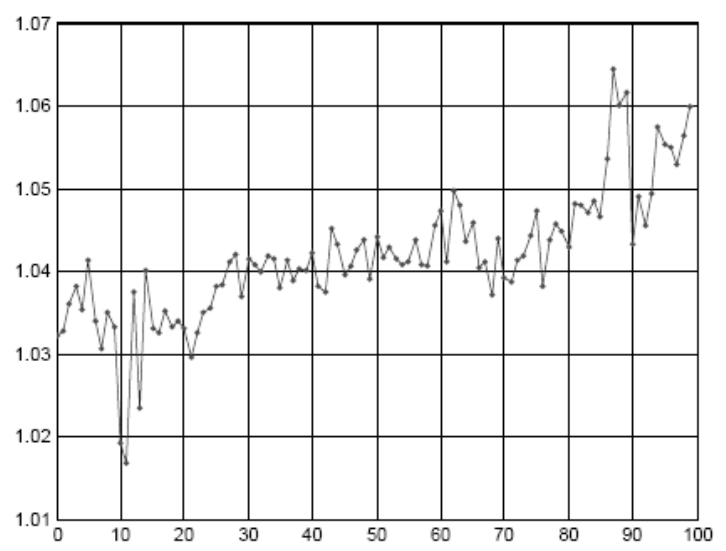

Figure 6. Fractal dimension change chart
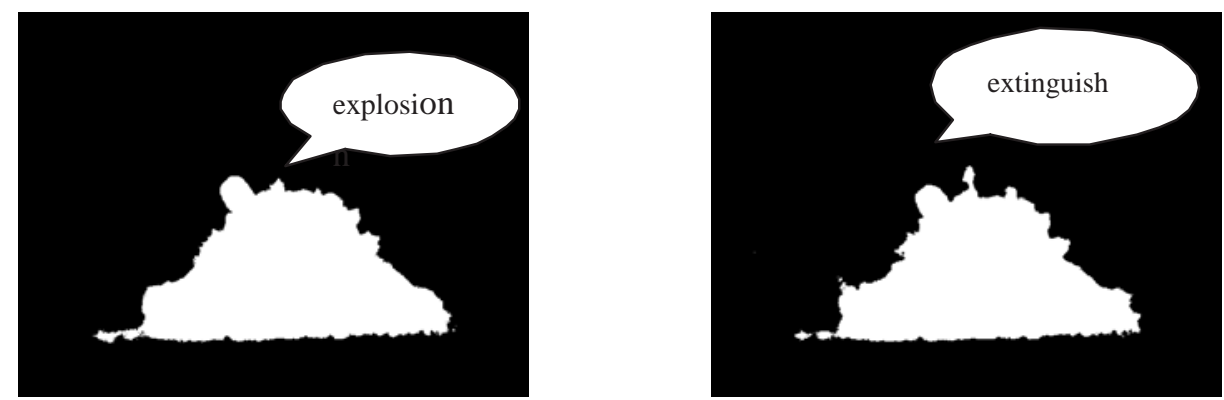

Dimension Value 1.0428

Dimension Value 1.0134

Figure 7. two value chart of detonation image in $3.5-4.5 \mathrm{~ms}$ 